\title{
A Survey of Different Approaches of Machine Learning in Healthcare Management System
}

\author{
Dr. Krishan Kumar Goyal \\ Associate professor, \\ Faculty of Computer Application, \\ Raja Balwant Singh Management Technical Campus, Agra. \\ E-Mail Id:kkgoyal@gmail.com \\ Aejaz Hassan Paray \\ Ph.D. Scholar Department of Computer Science, Bhagwant University Ajmer Rajasthan, \\ Aejaz50@gmail.com.
}

\begin{abstract}
Machine Learning has become an important tool in day to day life or we can say it's a powerful tool in most of the fields which we want to automate. Machine Learning is used to develop algorithms which can learn from the data, which is either labeled, unlabeled or learn from the environment. Machine Learning is used in most of the fields and Especially in health care sector it takes much more benefits through proper decision and prediction techniques. Machine Learning in health care is a scientific study, so we have to store, retrieve and proper use of information, data and provide knowledge to the problems facing in the healthcare sector and also knowledge for the proper decision making. Due to these technologies there is a huge development in health care sectors over the years. For analysis of medical data, medical experts use the machine learning tools and techniques to identify the risks and to provide proper diagnosis and treatment. The paper is based on survey in terms of health care management system using different machine learning approaches and techniques.
\end{abstract}

Keywords: Machine learning, Healthcare Management system, Artificial intelligence.

Date of Submission: Oct 10, 2019

Date of Acceptance: Dec 19, 2019

\section{Introduction}

$\mathrm{M}$ which aims to create intelligent system which do human like jobs by learning from a lot of relevant data. According to Nithya and Ilango[20], a computer program is said to learn from experiences $\mathrm{E}$ with respect to some classes of task $\mathrm{T}$ and performance measure $\mathrm{p}$, if its performance at task $\mathrm{t}$, as measured by $\mathrm{p}$ improves with experience $\mathrm{E}$. we can say a general algorithm takes the data and can learn automatically itself. In other words, we can say machine learning is computer ability to learn without being explicitly programming. Instead of writing a program by hand for each specific task, we can collect lot of examples or experiences(data) belonging to the problem, based on the data apply some classifiers do some processes and produces the output. The healthcare sector is being transformed by the ability to record massive amounts of information about individual patients, the enormous volume of data being collected is impossible for human beings to analyze. Machine learning provides a way to automatically find patterns and reason about data, which enables healthcare professionals to move to personalized care known as precision medicine. The purpose of the Classification model is to determine a label or category it is either one thing or another. We train the model using a set of labeled data. Machine learning is one of the emerging approaches in the health sector. As increasing population of patients the machine learning and Artificial intelligence will allow health care systems to make care more efficiently and appropriately .With the help of machine learning methods/approaches we identify the patients and provide proper care management. Patients can be identified (eg on the basis of patient's characteristics, risks, beliefs and genetic profile. Machine learning and Artificial Intelligence changing healthcare by using predictive analytics for proper treatment and decision without any risk factor. Machine learning and Artificial intelligence have been recently covers all the fields of healthcare services even though computers will completely replace doctors, nurses and modern technology. Recently Google develop a machine learning algorithm to identify the cancerous tumors and deep learning is used to identify the skin cancer. Machine Learning used to analyze the Enormous data and suggested outcomes, Risk factors and allocate the precise resources.

This paper serves many applications and techniques of machine learning in healthcare system. Rest of the paper is designed in following manner. In Section-II Supervised and Unsupervised learning techniques which are used in different health care management system are described. Section-III contains Reinforcement Learning Techniques in health care management system. Section-IV covers some of AI techniques used in health care management system. Finally Section-V concludes the paper. 


\section{Supervised and Unsupervised Machine Learning techniques in health care management system.}

As per Chuang et al. [21] there are seven well known supervised learning techniques namely C4.5, C5.0, CART(classification and regression tree), Logistic regression, SVM(support vector machine), Random forest, Multivariate adaptive regression splines. The C4.5 and CART are the two most commonly used decision treebased learning techniques. These techniques are used to develop a predictive model that determines the length of stay of patient in the hospital. The case was divided into urgent operation and non-urgent operation group to develop a prolonged length of stay prediction model using several supervised learning techniques. Critical factors are identified using the gain ratio technique contains medical records, lab data of 896 clinical cases involving surgeries performed by general physicians. Supervised learning technique is suitable for analyzing patient medical record in accurately predicting a prolonged length of stay. The proposed model supports a physician in making clinical decision and communicating with medical team members, patients and patient family. The system may also use for making decision regarding whether patient require more clinical care (therefore improving patient safety). Based on length of stay related literature, 119 independent variables are collected from laboratory information system, nursing information system and from physician orders then selected variables are divided into six categories. Demographic, Medical history, vital signs, laboratory data, operation physician data and operation \& nursing data. WEKA(version 36.6) an open source data mining program was used to execute variables. WEKA was used to construct a length of stay prediction model using several classification techniques. Some limitations of the present study are, First, the data used in this study were related to general surgery patients from a single medical institution. Proceeding with the evaluations of clinical cases from other hospitals is critical for confirming the validity of the model. Second, other potentially valuable features, such as data collected from the clinical pathway and supervised learning techniques, can be considered for use in the model. Finally, an interventional study may be initiated based on our finding in practicing an expert system that may be conducted in future studies and try to predict which patients will require urgent surgery. Shameer et al. [16] developed to predict readmission rates in heart failure patients. Readmission rates is a quality assessment used to improve the quality of life index of patient population and the quality of healthcare delivery. A machine learning is used for the generation of composite model for performing predictions using feature selected from individual model. In his study a naïve bayes model is used for machine learning. Bayesian model were created using feature unique to each data element and validation of machine learning models was performed with logistic regression. In the study we use multistep modeling strategy using Naïve bayes algorithm. In the first step, an individual model is created to classify the cases (readmitted) and controls (non-readmitted). In the second step, features contributing to predictive risk from independent models were combined into a composite model using a correlation-based feature selection (CFS) method. Data extracted from EMR: I) Diagnoses codes were extracted from Mount Sinai Data Warehouse. II) Medications prescribed during the hospitalization were extracted from Mount Sinai Data warehouse. Medication name, dosage, route of administration was obtained. III) Laboratory measurements. IV) Vital signs Pulse, respiration rate, systolic blood pressure, heartbeats and temperature were compiled and captured in a MySQL database. The research on CAD(computer aided diagnosis) and intelligent patient management system using different Machine learning and operations research techniques is proposed by Florin Gorunescu [11]. CAD is used for automated diagnosis of different major diseases, such as breast, pancreatic and lung cancer, heart attacks, diabetes using different supervised machine learning techniques (neural networks, support vector machines, Bayesian decision, $k$-nearest neighbor, etc) An intelligent patient management system develops an Application to patient grouping using different unsupervised machine learning techniques( $k$-means clustering) and Application to hospital bed management by optimal bed allocation, in accordance with the population needs and quality medical service. One can model a hospital/clinical department by using both queuing models and optimization tools $(\mathrm{M} / \mathrm{PH} / c$ and Genetic Algorithm). Nguyen et al. [17] proposed a remote health monitoring systems based on Internet of Things (IoT) technology. This paper explores the use of IoT-based applications in medical field and proposes an IoT Tiered Architecture (IoTTA) towards an approach for transforming sensor data into real-time clinical feedback. The IoT Tiered Architecture is based on the sensing, sending, processing, storing, mining and machine learning. Data mining involves discovering useful patterns from large data sets and applying algorithms to the extraction of hidden information. Its functions include classification, clustering, association analysis, time series analysis, and outlier analysis. Machine learning techniques are very useful in healthcare applications as they enable managing huge databases, learn from data and improve through experience (supervised as well as unsupervised). Alhamid et al. [7] proposed an open software framework that provides long-term monitoring of the patient's health situation by building a health monitoring system. That detecting any abnormality in the patient's health situation and particularly during their time spent at home. The other main objective is the development of a convenient method of analyzing and presenting the physiological data collected from a Wireless Body Sensor Network (WBSN). BSN consists of wearable sensors that include Bluetooth for communication. The paper also focus on the monitoring of Activity of Daily Living (ADL), which includes fall detection, walking, sitting, standing, and lying down as well as physiological monitoring such as heart rate using (simple activity recognition algorithm based on accelerometers signals, $\mathrm{K}$-nearest neighbor (KNN) classifier with an applied Bayesian Network. The 
researchers Madhumita Kathuria and Sapna Gambhir[8] focus on the E-health based on the Wireless Body Area Network (WBAN). WABN becomes an integral component of healthcare management system where a patient needs to be monitors both inside and outside home or hospital. The applications is categorized into: Retrieval, Alerting, Prediction, Suggestion and Reminders to health related information using machine learning techniques such as Binary Decision Tree and Genetic Algorithm. Decision tree is used to classify the unseen instances from the training set of pre-labeled instance. GA is applied on training sets to generate optimized training data sets. It is responsible for managing traffic flows at different levels so that both real time traffic and non-real time health related traffic get benefit. It is responsible to manage four kinds of works: Packet classification, queuing, scheduling and dropping. The researcher Lih-Jen Kau and ChihSheng Chen[13] proposed a smart phone based monitoring and rescue system. The smart phone should be android based only. The various wearable sensors are used for detecting patient if the accident occurs. The proposed system contains the triaxial accelerometer and gyroscope sensors. The triaxial accelerometer and electronic compass is uses as input if the fall detection occurs on smart phones. In order to process the input signal, system uses the cascading classifier and support vector machine (supervised learning) as detection tools. Once a fall accident event is detected, the user's position can be acquired by the global positioning system (GPS) or the assisted GPS (A-GPS), and sent to the rescue center via the $3 \mathrm{G}$ communication network so that the user can get medical help immediately. He Jian and $\mathrm{Hu}$ Chen [24] proposed a system that detects the fall detection of elderly. The system consists of custom vast and smart phone. The custom vast contains wearable motion detection sensor integrated with tri-axial accelerometer, gyroscope and Bluetooth. The tri-axial accelerometer, gyroscope is used to measure the reluctant acceleration and angular velocity that vary real-timely, and then make up stream data. Bluetooth is introduced to receive the stream data from sensors, Bluetooth is used to send the data to the smart phone. After getting the data the individual is falling or not based on the k-NN algorithm (supervised machine learning algorithm). The phone can make a call or send a message with GPS position to a healthcare center or family member as soon as it detects a fall. This system can provide a timely warning that a fall has occurred. The software includes the on-chip program running on the sensor board and the fall detection program app downloaded onto the mobile smart phone. Mshali et al. [22] surveyed about health monitoring system in smart environment. The aim of HMS is to not only reduce costs but to also provide timely e-health services to individuals wishing to maintain their independence. The most important functions and services offered by HMS for monitoring and detecting human behavior including its concepts, approaches, and processing techniques. The HMS with intelligent technologies, such as sensors (Personal Sensor Network, Body Sensor Network, Multimedia Devices) have resulted in a rapid emergence of smart environments. One such example is the so-called Health Smart Home (HSH). They have the potential to provide e-health services to meet the needs of this growing population. The machine learning methods and algorithms (supervised and unsupervised) to effectively interpret sensor data (low-level) and build new abstractions (highlevel) in order to understand this complex and variable data on human behavior. The methods and techniques have been widely used to recognize human behaviors, detect normal and abnormal situations and to predict future health conditions. We broadly classify techniques into three categories: probabilistic and statistical techniques, computational intelligence techniques, and knowledge-driven techniques.

1. Behavior Recognition: Statistical Techniques (Hidden Markov Models, Bayesian Network Naive Bayes, Multiclass Logistic Regression) Computational Intelligence Techniques (Neural Networks Support Vector Machine Decision tree (C4.5), Clustering) Knowledge-Driven Techniques in (Rule-based ,Fuzzy logic)

2. Behavior Abnormality Detection: Statistical Techniques (Gaussian Mixture Model, Hidden Markov Model) Computational Intelligence Techniques (Neural Networks, Support Vector Machine, Clustering ) Knowledge-Driven Techniques (Fuzzy logic, Ontologies )

3. Prediction: Statistical Techniques (Hidden Markov Models Computational Intelligence Techniques) Neural Networks (Support Vector Machine, Data Mining Techniques) Knowledge-Driven Techniques (Fuzzy logic). Mazhar et al. [18] developed a system using machine learning algorithms to better care and to ensure better facilities to the inpatients to predict Spinal Cord Injured (SCI) patient's length of stay. If we can predict accurate length of stay, patients do not have to leave in between the treatment without medical advice. This study proposes the use of multiple linear regressions. which will help better patient care in the future and help the hospital for the better resource management. Baker et al. [19] proposed the web portal to support a patient healthcare decision. The portal is powered by semantics open source software (SEMOSS) and end-to-end analytic tool. Multiple linear regression and random forest analysis is used in order to determine whether a patient would recommend a hospital on the basis of patient experience. The portal allows patient to search for and compare doctors and hospitals based on their personal needs. In this study the doctor's data used for analysis was obtained from the virgina board of medicine website. The data set includes 20 attributes for 40,0000 doctors attributes includes Name, Number of years in experience, sex etc. The distance is calculated using Google maps API. Hospital data include communicate with doctors, communication with nurses, responsiveness of hospital staff, pain management, communication about medicines, discharge information, cleanliness of hospital environment etc. Patient can check the doctor's data in their area and see the visualization of practitioners clustered by specialty. This type of graphic allows the patient to see how much choices they have when choosing a doctor of a certain specialty. The 
Mockup of the portal includes a home screen, search page, and various methods for viewing search results and visualization. Methods that are used for modeling a hospital data is multiple linear regression. Random forest analysis of hospital group.

\section{Reinforcement learning in health care management system.}

Niyato et al. [5] proposed A Remote patient monitoring system in terms of e-health service A patient-attached monitoring device with a heterogeneous wireless transceiver (WiMAX based WMAN and WiFi-based WLAN technologies) collects bio signal data from the sensors and transmits the data through the radio access network (RAN) to the eHealth service provider. The proposed architecture along with the reinforcement machine learning techniques (stochastic programming Problem, constrained Markov decision process (CMDP)) will be useful for an eHealth service provider to minimize the service cost while maintaining the quality-of-service (QoS) requirements for remote and mobile patient monitoring.

Due to the mobility of the patients, a stochastic programming problem has been formulated to obtain the optimal number of reserved connections and minimize the connection cost and satisfy the delay requirements. A constrained Markov decision process formulation has been used to obtain the optimal transmission scheduling decision.

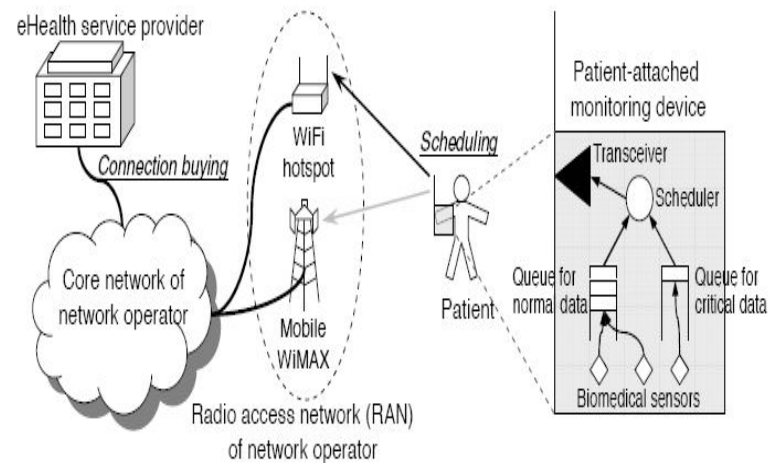

Fig 1. A mobile patient can use different types of wireless technologies (e.g., WiMAXbased WMAN and WiFi-based WLAN technologies) to transfer monitored bio signal data to the healthcare center.

Gaweda et al. [3] proposed the reinforcement machine learning methods for the treatment of various health relate problems in terms of markov decision processes. MDP is used to solve the stochastic and dynamic treatment decisions. An MDP binds previous, current, and future system decisions through the proper definition of system states. In order to provide the optimal decision in terms of health care MDP provides the various methods like Finitehorizon MDPs, Infinite-horizon MDPs (These two model provides an exact description of the system), SemiMarkov decision processes is used In health care and other applications, decisions may occur over continuous time intervals, such as when varying treatments can be administered. Finally, more hospitals are using electronic medical record systems to gather large amounts of patient data. While using this data will open the door for the increased application of MDPs to medical treatment problems. Hauskrecht and Fraser [1] proposed a method of Markov decision processes viz partially observable Markov decision processes for the treatment of ischemic heart disease. A disease caused by an imbalance between the supply and demand of oxygen to the heart. The proposed system uses methods for the treatment of ischemic heart disease, characterized by hidden disease states, investigative and treatment procedures, and temporal cost and their outcomes. Khianjoom and Wipawee [9] Monitoring the patients by transferring the diagnostic information if the patient fall. They presents an efficient, adaptive, distributed routing mechanism using Anycast Q-routing to route information to the nearest sink. Anycast has been used in wired networks and mobile ad hoc networks, to select the nearest server, service identification, improve system reliability and policy routing. Q-routing is based on Q-learning which is a wellknown Reinforcement Learning method. RL is a machine learning technique which aims at finding the optimal action to perform at a given state of the dynamic environment. The proposed scheme requires low communication overhead and fast path search time to discover optimal paths to the nearest sink. Gaweda et al. [2] proposed the system which provides an optimal treatment to the patient of anemia using reinforcement learning approach. The learning for the optimal treatment occurs in the form of immediate improvements in the drug dosing policy due to the experience gained by observing i.e. off -policy using Q-learning rather than on-policy that is learning form processing. Q-learning is capable of performing adequate anemia treatment in real time. To classify the different types of patients, a Takagi-Sugeno fuzzy model was first built on basis of available patient data.

\section{Some AI techniques used in the health care management system.}

Hurwitz et al. [10] proposed cognitive computing, which is combination of cognitive science and computer science. cognitive computing represents self-learning system that utilizes machine learning model to mimic the way brain works. A cognitive computing is used by combining technologies such as machine learning, artificial intelligence and natural language processing, that helps healthcare professionals to learn from patterns which means healthcare organizations to solve some of their most challenging problems. For example, The University of Iowa Hospitals and Clinics has identified patterns in a population of surgical patients that help to improve both quality and performance in surgery. The hospital has modeled data from hospital readmission, surgical site infections, and other hospital acquired infections. The model enables physicians to predict which patients are most at risk for acquiring a surgical site infection while 
they are still in the operating room and corrective actions can be taken. Cognitive system enables healthcare organization to gain more value from data. Gaining more value from data is a multifaceted process that requires both technology and human knowledge. Getting the data right is more important. The relevant data needs to be accurate, trusted, consistent, and available for access. However, having accurate data is only the baseline for improving health outcomes for patients. Physicians need the skill and experience to make sense out of what is often a complex set of symptoms and diagnostic tests. They need to internalize best practices that enable them to ask the right questions and listen for answers from the patient. A cognitive system helps in find patterns and outliers in data that can help to fast track new treatments, improve efficiencies, and treat patients more effectively. But the limitation of cognitive computing is that the system fails at analyzing the risk which is missing in unstructured data. That includes socio-economic factors, culture, political environment and people. The scope of cognitive technology is limited to engagement and decision.

In Altintop et al. [14] healthcare management, they tried to provide quality healthcare service and the effective utilization of limited resources, it is necessary to make efficiency measurement or knowledge discoveries on raw data in the Healthcare information system. This can be done using two methods. The first is the efficiency measurement techniques and data mining techniques. In this study, fuzzy linguistic summarization is used to analyze operational and financial data of the healthcare facilities. Fuzzy linguistic summarization has been shown to be simple, efficient and human consistent way of knowledge extraction. The linguistic summaries are kind of outputs of Decision support systems (DSS). Decision support systems (DSS) are a class of computerized information system that support decision-making activities. The main idea in linguistic summarization for the healthcare is to provide the statements such as "Most hospitals with few beds have a small amount of total budget. There are two approaches for generating fuzzy linguistic summaries. One way is to generate all possible linguistic summaries and select them, of which truth degree is over a predefined threshold. However, this way is very exhaustible when the number of summarizers becomes very large. The other way to generate fuzzy linguistic summaries is to use metaheuristics such as genetic algorithms. The idea behind the use of genetic algorithm is that the individuals (summaries) of which truth degree are equal or greater than the predefined threshold will only survive after several generation evolutions. This makes that a desirable set of linguistic summaries can be efficiently found instead of generating all linguistic summaries. In this paper genetic algorithm is used to generate fuzzy linguistic summaries. In this study, the dataset of Operational and financial healthcare covering the records for the year 2013, which belong to 677 public hospitals located in Turkey with 227 features that are categorized into 3 groups, 1. Administrative which contains Total number of medical examination, Load factor of beds, Total number of inpatients, City population, etc. 2. Income Items which contains Total budget, Basic salary expense, Social right expense, Medical device expense, etc. and 3. Expense Items contains Medication income, Inpatient medical implementation income, Outpatient laboratory income, Social security institution income, etc. is used. The provinces where hospitals are located are also classified. A genetic algorithm is developed to generate fuzzy linguistic summaries. In this system, the parameters of genetic algorithms were established with trial and error for the presented operational and financial healthcare dataset. The proposed approach is implemented in Java environment .linguistic summarization is meant as a process of a comprehensive description of big and complex datasets through short statements in natural language .but the limitation of this approach is representation and processing of imprecision that is characteristic for natural language. Roderick et al. [15] used Data science in health care, Description of medical data, Machine learning in health care, and Prediction of adverse events based on the traditional medical data. Data science is defined as a process of extracting knowledge from externally generated data. It is combination of mathematics, statistics, computer science and applied field of study. Description of medical data by storing and analyzing medical histories for millions of patients, using thousands of variables to provide better resource allocations. The stored healthcare data is provide as a input into the model in order to make predictive model for rare events. In this study the health care data are in terms of : (a) Census information (such as age or ethnicity) and simplest biometrics (such as weight). (b) Clinical information: time stamped labels of transactions, assigned according to a medical coding system. These record instances of medical decisions, not detailed outcomes. (c) Financial information: depending on conditions of data sharing with insurance providers, this may include claims data, payments received and aggregate metrics of subscriber loyalty or risk. (d) Narratives, including unstructured text documents such as doctor's notes. (e) Multiformatted measurements and test results, including numerical data and images. (f) Self-reported behavior, such as questionnaires of patient satisfaction. (g) Inferred and miscellaneous socioeconomic features, such as frequency of use of social media or likelihood to purchase an insurance plan. Machine learning methods (supervised and unsupervised algorithms ) are used for medical data which is noisy, imbalanced, standard predictors and classifiers makes the data proper and clear. Machine learning take a model in cognition process in that it deals with patterns. It decomposes and synthesizes information. Decomposition leads to creation of patterns that will be recognized when observed again. Synthesis allows completion of partially observed data into familiar patterns. Supervised learning aims at achieving the best representation of output. Unsupervised learning attempts to best group subsets among different sets of data(input). Lastly the paper predicts rare adverse events for general patient population, based on nonspecific, traditional clinical data, identified clinical and medical insurance records of 500,000 patients. This study is performed in the 
framework of personalized medicine that automatically select only the most relevant training data for each patient, but at the same time don't want to restrict the study to any particular population group such as in-patients, or patients already recorded as having increased risk of an adverse event. This has led us to a decision to build an individual predictive model for each patient. In Khalaf et al. [12], system reduces costs, provide 24 hours communication between medical experts and patients and remote monitoring for SCD patients. When the system detects any critical condition from the patient, it generates an automatic message to the medical doctors based on webbased platform in order to assist them with optimal decision-making using ANN with MIP algorithm is to extract the information from medical dataset automatically and supervised machine learning algorithms for the prediction of the amount of medication. The proposed framework is divided into two sides. The patient side is used to monitor, to store and collect data, as well as to send notifications to the clinicians in terms of abnormal conditions. The hospital side consists of a database and a decision support system. Medjahed et al. [6] proposed an automatic in-home healthcare telemonitoring system for several use. The proposed telemonitoring system is a multimodal platform with several sensors that can be installed at home. A set of microphones placed in all rooms, for allow remote monitoring. wearable device called RFpat that can measure physiological data like ambulatory pulse heart rate, detect posture (standing/ sitting and laying). A set of infrared sensors that detect the person's presence in a portion of a given home also his movement and a set of domotic sensors like contact sensors, temperature sensors and several other domotic sensors for environment conditions monitoring. With the help of these sensors is the only way to obtain the required breadth of information. But the key to produce the required detection is to use the right method that properly fuses the provided data from various sources called multimodal data fusion. The fuzzy logic method is the proposed way to meet these challenges of this multimodal data fusion application. The fuzzy logic decision module reinforces the secure detection of older person distress events and his localization. Miškuf et al. [23] proposed a cloud-based architecture for the healthcare data classification allows to create one smart system to monitor, track, and store patients healthcare information for ongoing care. Therefore, we provide our case study in which we took hospital data and used cloud services for data storing and machine learning. Then we have developed a web application on the top of this architecture and evaluated the concept in the real environment of the hospital. The keywords used in this paper is Healthcare IoT, Data Mining, Azure ML, R Shiny. The aim of this paper is to present the concept definition of a platform for HealthI IoT that connects the advantages of IoT with the emerging cognitive computing. The healthcare Industrial Internet of Things (HealthIIoT) is a combination of communication technologies, things, applications, and people that would function together as one smart system to monitor, track, and store patients healthcare information for ongoing care. Lee et al. [4] proposed a conceptual classification model toward consumer's behavior in choosing hospital, Besides, the back propagation network was utilized to build the classification model for finding consumers' behavior of choosing hospitals. A neural network model is useful in identifying existing patterns of hospital's consumers.

\section{Conclusion:}

In this study, we provide the comprehensive survey of Machine learning approaches in terms of heath care management system. we focus on how the different methods of machine learning whether they are supervised, unsupervised or Reinforcement are used for different healthcare problems. The healthcare problem can be: fast treatment of a patient, prediction of various diseases, Remotely Monitoring the Patients using internet of things (IOT) and Monitoring the patient using sensors.

\section{References:}

1. Hauskrecht, Milos, and Hamish Fraser. "Planning treatment of ischemic heart disease with partially observable Markov decision processes." Artificial Intelligence in Medicine 18.3 (2000): 221-244.

2. Gaweda, Adam E., et al. "Individualization of pharmacological anemia management using reinforcement learning." Neural Networks 18.5-6 (2005): 826-834.

3. Schaefer, Andrew J., et al. "Modeling medical treatment using Markov decision processes." Operations research and health care. Springer, Boston, MA, 2005. 593-612.

4. Lee, Wan-I., Bih-Yaw Shih, and Yi-Shun Chung. "The exploration of consumers' behavior in choosing hospital by the application of neural network." Expert systems with applications 34.2 (2008): 806-816.

5. Niyato, Dusit, Ekram Hossain, and Sergio Camorlinga. "Remote patient monitoring service using heterogeneous wireless access networks: architecture and optimization." IEEE Journal on Selected Areas in Communications 27.4 (2009).

6. Medjahed, Hamid, et al. "A pervasive multi-sensor data fusion for smart home healthcare monitoring." Fuzzy Systems (FUZZ), 2011 IEEE International Conference on. IEEE, 2011.

7. Alhamid, Mohammed F., et al. "Hamon: An activity recognition framework for health monitoring support at home." Instrumentation and Measurement Technology Conference (I2MTC), 2011 IEEE. IEEE, 2011.

8. Kathuria, Madhumita, and Sapna Gambhir. "Leveraging machine learning for optimize predictive classification and scheduling E-Health traffic." Recent Advances and Innovations in Engineering (ICRAIE), 2014. IEEE, 2014.

9. Khianjoom, Siwatchaya, and Wipawee Usaha. "Anycast Q-routing in wireless sensor networks for healthcare monitoring." Electrical Engineering/Electronics, 
Computer, Telecommunications and Information Technology (ECTI-CON), 2014 11th International Conference on. IEEE, 2014.

10. Hurwitz, Judith S., Marcia Kaufman, and Adrian Bowles. Cognitive computing and big data analytics. John Wiley \& Sons, 2015.

11. Gorunescu, Florin. "Intelligent decision systems in Medicine-A short survey on medical diagnosis and patient management." E-Health and Bioengineering Conference (EHB), 2015. IEEE, 2015.

12. Khalaf, Mohammed, et al. "Applied Difference Techniques of Machine Learning Algorithm and WebBased Management System for Sickle Cell Disease." Developments of E-Systems Engineering (DeSE), 2015 International Conference on. IEEE, 2015.

13. Kau, Lih-Jen, and Chih-Sheng Chen. "A smart phonebased pocket fall accident detection, positioning, and rescue system." IEEE journal of biomedical and health informatics19.1 (2015): 44-56.

14. Altintop, Tunahan, et al. "Fuzzy Linguistic Summarization with Genetic Algorithm: An Application with Operational and Financial Healthcare Data." International Journal of Uncertainty, Fuzziness and Knowledge-Based Systems 25.04 (2017): 599-620.

15. Roderick, Oleg, et al. "DATA ANALYSIS AND MACHINE LEARNING EFFORT IN HEALTHCARE: ORGANIZATION, LIMITATIONS, AND DEVELOPMENT OF AN APPROACH." Internet of Things and Data Analytics Handbook (2017): 295-328.

16. Shameer, Khader, et al. "Predictive modeling of hospital readmission rates using electronic medical recordwide machine learning: a case-study using Mount Sinai Heart Failure Cohort." PACIFIC SYMPOSIUM ON BIOCOMPUTING 2017.

17. Nguyen, Hoa Hong, et al. "A review on IoT healthcare monitoring applications and a vision for transforming sensor data into real-time clinical feedback." Computer Supported Cooperative Work in Design (CSCWD), 2017 IEEE 21st International Conference on. IEEE, 2017.

18. Mazhar, Tabib Ibne, et al. "Spinal Cord Injured (SCI) patients' length of stay (LOS) prediction based on hospital admission data." Electrical Information and Communication Technology (EICT), 2017 3rd International Conference on. IEEE, 2017.

19. Baker, Claire, et al. "Healthcare analytics and visualization using SEMantic Open Source Software (SEMOSS)." Systems and Information Engineering Design Symposium (SIEDS), 2017. IEEE, 2017.

20. Nithya, B., and V. Ilango. "Predictive analytics in health care using machine learning tools and techniques." International Conference on Intelligent Computing and Control Systems (ICICCS). IEEE, 2017.
21. Chuang, Mao-Te, Ya-han Hu, and Chia-Lun Lo. "Predicting the prolonged length of stay of general surgery patients: a supervised learning approach." International Transactions in Operational Research 25.1 (2018): 75-90.

22. Mshali, Haider, et al. "A survey on health monitoring systems for health smart homes." International Journal of Industrial Ergonomics 66 (2018): 26-56.

23. Miškuf, Martin, Iveta Zolotová, and Jozef Mocnej. "Healthcare data classification-Cloud-based architecture concept." Cybernetics \& Informatics (K\&I), 2018. IEEE, 2018.

24.Jian, $\mathrm{He}$, and $\mathrm{Hu}$ Chen. "A portable fall detection and alerting system based on $\mathrm{k}-\mathrm{NN}$ algorithm and remote medicine." China Communications 12.4 (2018): 23-31.

\section{Profile}

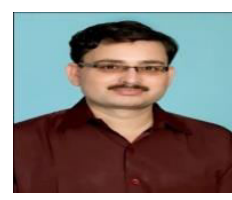

Dr. Krishan Kumar Goyal received M.Tech in Computer science from U.P. Technical University, Lucknow. He has received $\mathrm{Ph} . \mathrm{D}$. in cryptography from Dr. B. R. Ambedkar University, Agra. He has also received master's degree in Computer Application \& Mathematics from Dr. B.R. Ambedkar University, Agra. Presently, he is working as an Associate Professor and Dean, Faculty of Computer Application at Raja Balwant Singh Management Technical Campus, Agra. He has participated in several faculty development programs, seminars and workshops. He has published several research papers in leading journals of national and international repute. He has authored two books. He is Life member of several societies such as Computer Society of India, Ramanujan Mathematical Society of India, Cryptology Research Society of India etc. He is also a reviewer and member of editorial board of different national and international journals. His area of interest includes Cryptography, Cyber Security, Privacy and Security in Online Social Media, Machine Learning, Natural Language Processing etc.

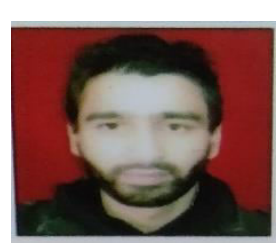

Aejaz Hassan Paray received Master of Computer Application from University of Kashmir. Presently he is pursing his Ph.D degree in Computer Application from Bhagwant University of Ajmer Rajasthan. He has participated in several faculty development programs, seminars and workshops. He has published several research papers in leading journals of national and international repute. He is member of several societies such as Computer Society of India etc. His area of interest includes Artificial Intelligence, Artificial Neural Network, Machine Learning etc. 